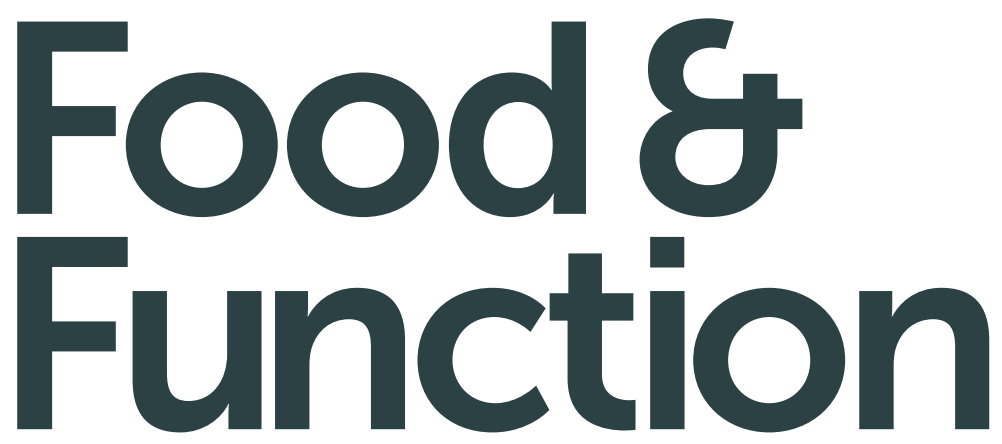

Linking the chemistry and physics of food with health and nutrition www.rsc.org/foodfunction
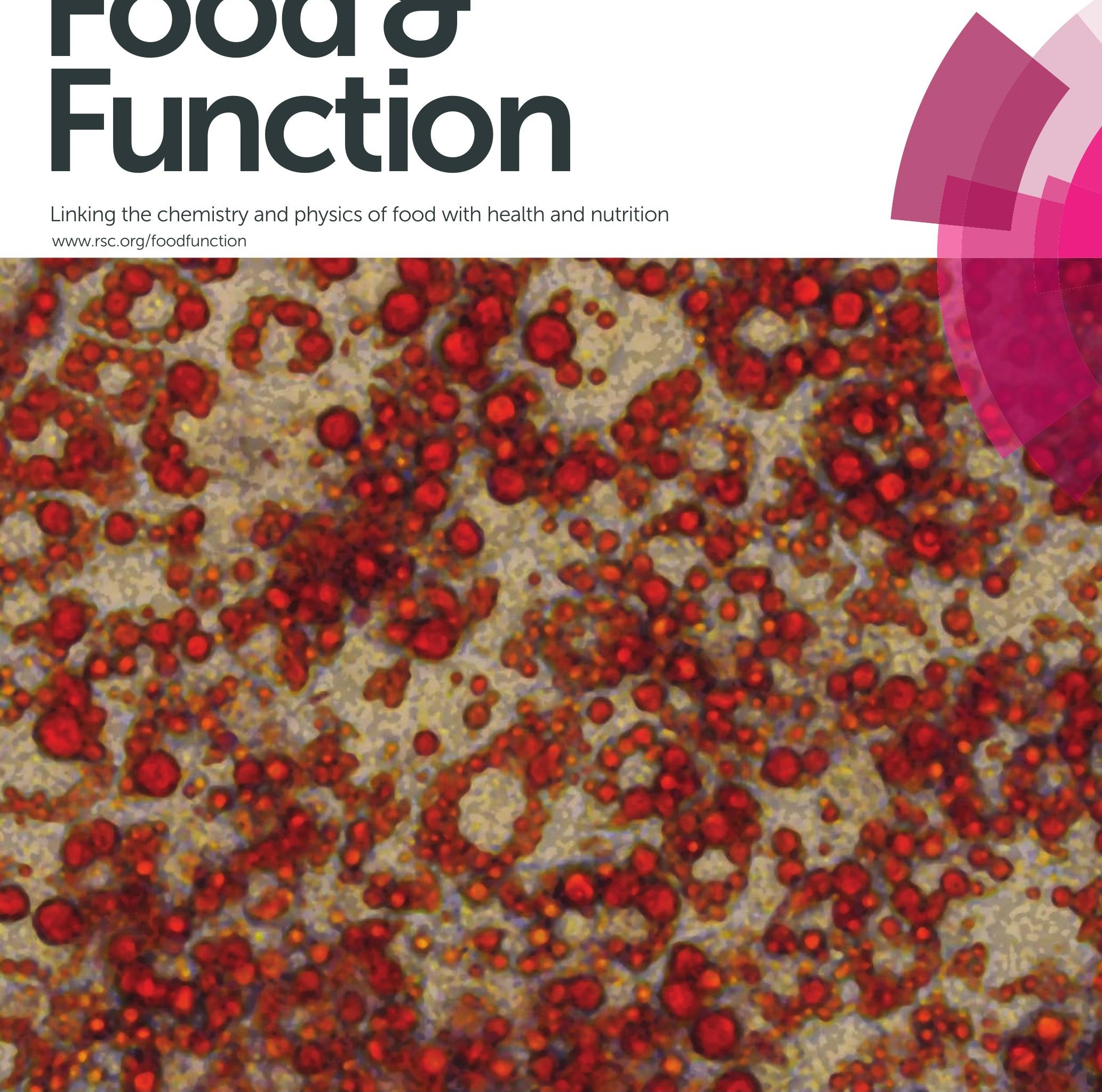

ISSN 2042-6496

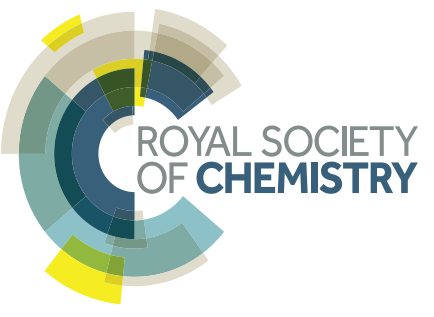




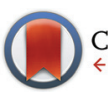

CrossMark \& click for updates

Cite this: Food Funct., 2015, 6, 2135

\title{
Polyacetylenes from carrots (Daucus carota) improve glucose uptake in vitro in adipocytes and myotubes
}

\author{
Rime B. El-Houri, $\dagger^{\mathrm{a}}$ Dorota Kotowska, $\dagger^{\mathrm{b}}$ Kathrine B. Christensen, ${ }^{\mathrm{a}}$ \\ Sumangala Bhattacharya, ${ }^{c}$ Niels Oksbjerg, ${ }^{C}$ Gerhard Wolber, ${ }^{d}$ Karsten Kristiansen ${ }^{b}$ \\ and Lars P. Christensen*a
}

A dichloromethane (DCM) extract of carrot roots was found to stimulate insulin-dependent glucose uptake (GU) in adipocytes in a dose dependent manner. Bioassay-guided fractionation of the DCM extract resulted in the isolation of the polyacetylenes falcarinol and falcarindiol. Both polyacetylenes were able to significantly stimulate basal and/or insulin-dependent GU in 3T3-L1 adipocytes and porcine myotube cell cultures in a dose-dependent manner. Falcarindiol increased peroxisome proliferator-activated receptor (PPAR) $\gamma$-mediated transactivation significantly at concentrations of 3,10 and $30 \mu \mathrm{M}$, while PPAR $\gamma$ mediated transactivation by falcarinol was only observed at $10 \mu \mathrm{M}$. Docking studies accordingly indicated that falcarindiol binds to the ligand binding domain of PPAR $\gamma$ with higher affinity than falcarinol and that both polyacetylenes exhibit characteristics of PPAR $\gamma$ partial agonists. Falcarinol was shown to inhibit adipocyte differentiation as evident by gene expression studies and Oil Red $O$ staining, whereas falcarindiol did not inhibit adipocyte differentiation, which indicates that these polyacetylenes have distinct modes of action. The results of the present study suggest that falcarinol and falcarindiol may represent scaffolds for novel partial PPAR $\gamma$ agonists with possible antidiabetic properties.

Received 4th March 2015 Accepted 4th May 2015

DOI: $10.1039 / c 5 f o 00223 k$ www.rsc.org/foodfunction
T2D is currently treated with a combination of diet restriction and oral drugs, which include sulfonylureas, repaglinide, biguanidines (e.g., metformin), $\alpha$-glucosidase inhibitors, and thiazolidinediones (TZDs). These oral drugs exert their hypoglycemic effects through different mechanisms of action such as enhancement of insulin secretion by pancreatic $\beta$-cells, reduction of hepatic glucose production, improvement of insulin sensitivity, and inhibition of intestinal glucose digestion and absorption. ${ }^{5}$ TZDs such as rosiglitazone (Rosi) and pioglitazone are efficient insulin-sensitizing drugs. TZDs act by activating peroxisome proliferator-activated receptor (PPAR) $\gamma^{6,7}$ Activation of PPAR $\gamma$ leads to a conformational change in the ligand binding domain (LBD) and this process then alters the transcription of several target genes involved in carbohydrate and lipid metabolism resulting in for example facilitation of glucose and lipid uptake, decrease in free fatty acid levels and amelioration of insulin resistance. ${ }^{7-9}$ TZDs may cause severe side effects such as increased water retention, weight gain, heart enlargement, and hepatotoxicity; ${ }^{10}$ consequently, many TZDs have been withdrawn from the market. The unwanted side effects of TZDs have been linked to their behavior as full agonists of PPAR $\gamma .{ }^{6} \operatorname{PPAR} \gamma$ partial agonists on the other hand are compounds with diminished agonist efficacy that maintain the insulin sensitizing effect but 
without inducing the same magnitude of side effects as observed for full agonists. ${ }^{6}$ Molecular docking studies have revealed that full and partial agonists have different binding modes in the LBD of PPAR $\gamma \cdot{ }^{11,12}$ This may explain why full and partial agonists recruit different sets of co-activators, and exhibit different pharmacological activities. ${ }^{11,13}$

Plants have a long history in the traditional treatment of diabetes, and are therefore a promising source of natural products with potential antidiabetic effects and improved modes of action. ${ }^{14,15}$ Using a screening platform for identification of plant extracts with potential bioactivity related to insulindependent GU and fat accumulation we recently demonstrated that the dichloromethane (DCM) extract of carrot roots (Daucus carota, Apiaceae) is able to activate PPAR $\gamma$ and to stimulate insulin-dependent GU in 3T3-L1 adipocytes and porcine myotubes. ${ }^{16}$ The objective of the present investigation was therefore (i) to investigate the DCM extract for potential antidiabetic natural products by a bioassay-guided fractionation approach using insulin-dependent GU in 3T3-L1 adipocytes as the bioassay, and (ii) to investigate the antidiabetic potential of isolated natural products from active fractions in adipocytes and myotubes.

\section{Materials and methods}

\section{Chemicals and reagents}

Silica gel 60 aluminum sheets for TLC and Silica gel 60 (particle size $0.063-0.2 \mathrm{~mm}$ ) for flash column chromatography were purchased from Merck (Darmstadt, Germany). CHROMASOLV® HPLC grade ACN, ethyl acetate, methanol, DCM, $n$-hexane, isopropanol and ReagentPlus ${ }^{\circledR}$ grade of vanillin, sulfuric acid, TFA, and DMSO were purchased from SigmaAldrich (Steinheim, Germany). Water was purified with a MilliQ system consisting of an Ultra Clear Basic UV system (Holm \& Halby, Brøndby, Denmark). For adipocyte bioassays, DMEM, foetal calf serum (FCS), calf serum (CS), phosphate buffered saline (PBS), Krebs-Ringer-Hepes buffer (KRHB), $\left[{ }^{14} \mathrm{C}\right] 2$-deoxyD-glucose, $\quad N$-(2-hydroxyethyl)-piperazine-2'-(2-ethane-sulfonic acid) (HEPES), penicillin/streptomycin and TRIzol reagent were purchased from Life Technologies, Invitrogen A/S (Tåstrup, Denmark). Dexamethasone, 1-methyl-3-isobutylxanthine, insulin, Oil Red $\mathrm{O}$ and sodium dodecyl sulphate (SDS) were purchased from Sigma-Aldrich (Brøndby, Denmark). Rosiglitazone (Rosi) was purchased from Novo Nordisk A/S (Bagsvaerd, Denmark). For myotube cultures, FCS, horse serum, DMEM, and 2-deoxy-[ $\left.{ }^{3} \mathrm{H}\right]$-D-glucose were purchased from Life Technologies (Naperville, IL, USA). Matrigel matrix was purchased from BD Biosciences Denmark A/S (Albertslund, Denmark).

\section{Extraction and fractionation procedure and isolation of bioactive compounds}

Fresh carrot roots (cv. Bolero) were obtained from the Department of Food Science, Aarhus University, Denmark. The roots (3 kg) were homogenized and extracted twice with DCM (12 l) for $24 \mathrm{~h}$ in the dark at $5{ }^{\circ} \mathrm{C}$ with periodical shaking. The extracts were combined, filtered, and dried under vacuum $\left(30^{\circ} \mathrm{C}\right)$. The DCM extract $(10 \mathrm{~g})$ was separated by flash column chromatography (100 mm i.d., $300 \mathrm{~g}$ silica gel) by the following gradient: $100 \% n$-hexane $(600 \mathrm{ml}), 10-90 \%$ ethyl acetate in $n$-hexane in $10 \%$ steps (600 ml each), 100\% ACN (1000 ml). Seventy fractions (100 ml each) were collected and fractions with the same metabolite profile, based on TLC analysis were combined, resulting in 10 fractions (numbered A-J). TLC plates were developed with ethyl acetate $: n$-hexane $(30: 70)$ and metabolites were visualized by UV light and a vanillin-sulfuric acid reagent. Fractions A-J were all tested in the adipocyte GU bioassay resulting in the active fractions $\mathrm{C}(70 \mathrm{mg})$ and $\mathrm{F}$ (30 mg). Fraction $\mathrm{C}$ and $\mathrm{F}$ were further separated by semipreparative HPLC into 14 fractions (numbered C1-C14) and 9 fractions (numbered F1-F9), respectively, on a Dionex UltiMate 3000 Binary Semi-preparative LC System from Thermo Fisher Scientific (Hvidovre, Denmark), equipped with DAD, and a Foxy Jr. fraction collector unit from Teledyne ISCO Inc. (NE, USA). Separations were performed on a Develosil ODS-HG-5 reversed phase C18 column $(5 \mu \mathrm{m} ; 250 \times 20 \mathrm{~mm}$, Nomura Chemical Co., Japan). The mobile phase consisted of water containing $0.05 \%$ TFA (solvent A) and ACN containing $0.05 \%$ TFA (solvent B). The following gradient was used: $25 \% \mathrm{~B}$ at $0 \mathrm{~min} ; 100 \% \mathrm{~B}$ at $55 \mathrm{~min} ; 100 \% \mathrm{~B}$ at $75 \mathrm{~min} ; 25 \% \mathrm{~B}$ at $80 \mathrm{~min}$; $25 \%$ at $90 \mathrm{~min}$. Column temperature was $25{ }^{\circ} \mathrm{C}$, flow rate was $5 \mathrm{ml} \mathrm{min}{ }^{-1}$, and injection volume was $2 \mathrm{ml}$. Metabolites were monitored at 210 and $254 \mathrm{~nm}$, and UV spectra recorded between 200 and $600 \mathrm{~nm}$. Fractionation by semi-preparative HPLC resulted in two active fractions containing pure falcarinol (fraction C6, $10 \mathrm{mg}$, LC-DAD-MS purity >98\%) and falcarindiol (fraction F2, $13 \mathrm{mg}$, LC-DAD-MS purity >98\%).

\section{Characterization of the bioactive polyacetylenes falcarinol and falcarindiol}

Falcarinol and falcarindiol were identified by LC-DAD-APCI-MS, $1 \mathrm{D}$ and 2D NMR, and optical rotation. LC-MS data were generated on a LTQ XL (2D Linear Quadrupole Ion Trap, Thermo Scientific, USA) mass spectrometer operated in APCI positive mode and attached to an Accela HPLC pump and a DAD. Settings for the mass spectrometer were 50, 5, and 5 (arbitrary units) for sheath, auxiliary, and sweep gas flow rates $\left(\mathrm{N}_{2}\right)$, respectively, a vaporizer temperature of $450{ }^{\circ} \mathrm{C}$, a discharge current of $5 \mu \mathrm{A}$, a capillary temperature of $275^{\circ} \mathrm{C}$, a capillary voltage of $16 \mathrm{~V}$, and a tube lens of $35 \mathrm{~V}$. Falcarinol and falcarindiol were separated on a LiChrospher® 100 reversed phase C18 column $(5 \mu \mathrm{m} ; 250 \mathrm{~mm} \times 4.6 \mathrm{~mm}$, Merck Millipore A/S, Hellerup, Denmark). The mobile phase consisted of water containing $0.05 \%$ TFA (solvent A) and ACN containing $0.05 \%$ TFA (solvent $\mathrm{B}$ ). The following gradient was used: $25 \% \mathrm{~B}$ at $0 \mathrm{~min} ; 100 \% \mathrm{~B}$ at $25 \mathrm{~min} ; 100 \% \mathrm{~B}$ at $35 \mathrm{~min}$; $25 \% \mathrm{~B}$ at $36 \mathrm{~min} ; 25 \%$ at $40 \mathrm{~min}$. Column temperature was

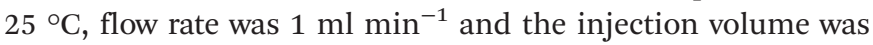
$10 \mu \mathrm{l}$. Compounds were monitored at 210 and $254 \mathrm{~nm}$, and UV spectra were recorded between 200 and $600 \mathrm{~nm}$. Falcarindiol and falcarinol eluted at retention time $19.9 \mathrm{~min}$ and $24.4 \mathrm{~min}$, 
respectively, with the following UV and MS data: falcarinol, $\lambda_{\max } 233,246,259 \mathrm{~nm} ; m / z 309\left[\mathrm{M}+\mathrm{H}-\mathrm{H}_{2} \mathrm{O}+2 \mathrm{ACN}\right]^{+}, 268$ $\left[\mathrm{M}+\mathrm{H}-\mathrm{H}_{2} \mathrm{O}+\mathrm{ACN}\right]^{+}, 245[\mathrm{M}+\mathrm{H}]^{+}, 227\left[\mathrm{M}+\mathrm{H}-\mathrm{H}_{2} \mathrm{O}\right]^{+}$; Falcarindiol, $\lambda_{\max } 231,244,257 \mathrm{~nm} ; m / z 284\left[\mathrm{M}+\mathrm{H}-\mathrm{H}_{2} \mathrm{O}+\right.$ $\mathrm{ACN}]^{+}, 261[\mathrm{M}+\mathrm{H}]^{+}, 243\left[\mathrm{M}+\mathrm{H}-\mathrm{H}_{2} \mathrm{O}\right]^{+}, 225[\mathrm{M}+\mathrm{H}-$ $\left.2 \mathrm{H}_{2} \mathrm{O}\right]^{+}$. Retention time, UV and MS data of falcarinol and falcarindiol were identical with those of authentic reference compounds obtained from previous research. ${ }^{17} \mathrm{NMR}$ data $\left({ }^{1} \mathrm{H},{ }^{13} \mathrm{C}\right.$, DEPT, COSY, and HSQC) were acquired on a Bruker $400 \mathrm{MHz}$ spectrometer in $\mathrm{CDCl}_{3}\left(\delta_{\mathrm{H}} 7.26 / \delta_{\mathrm{C}} 77.7\right)$ with tetramethylsilane as internal standard and optical rotation was recorded on an ADP440+ digital polarimeter (Bellingham + Stanley Ltd, UK). The complete spectroscopic and spectrometric data set of the isolated polyacetylenes were in accordance with literature values for (3R,9Z)-heptadeca-1,9-dien-4,6-diyn-3-ol (falcarinol) and $(3 R, 8 S, 9 Z)$-heptadeca-1,9-dien-4,6-diyn-3,8-diol (falcarindiol). ${ }^{18,19}$

\section{Preparation of samples for bioassays}

Crude extract, fractions, and isolated compounds, were dissolved in DMSO and stored at $-80{ }^{\circ} \mathrm{C}$ until testing in the bioassays. For testing in bioassays, the samples were dissolved in the testing media reaching a concentration of $0.1 \%(\mathrm{v} / \mathrm{v})$ DMSO and used as the stock solution.

\section{Adipocyte cell cultures}

3T3-L1 preadipocytes were cultured in DMEM with 10\% CS supplemented with $1 \%$ penicillin/streptomycin at $37{ }^{\circ} \mathrm{C}$ in humidified $95 \%$ air and $5 \% \mathrm{CO}_{2}$. At day two post-confluence (designated day 0 ) the cells were induced to differentiate with $500 \mu \mathrm{M}$ 1-methyl-3-isobutylxanthine, $1 \mu \mathrm{M}$ dexamethasone, and $167 \mathrm{nM}$ insulin in DMEM supplemented with 10\% FCS (MDI protocol). At day 2 the medium was replaced with DMEM supplemented with $10 \%$ FCS, $1 \%$ penicillin/streptomycin, and $167 \mathrm{nM}$ insulin. Thereafter medium was replaced every second day with DMEM supplemented with 10\% FCS and 1\% penicillin/streptomycin for both treatments. Extract, fractions, and pure compounds were dissolved in $0.1 \%$ DMSO and thereafter added to the medium to a final concentration as indicated. Vehicle cells were treated with $0.1 \%$ DMSO equal to the DMSO concentration in medium containing test samples.

\section{GU in 3T3-L1 adipocytes}

Mature 3T3-L1 cells were seeded in 96-well plates and differentiated according to the MDI protocol till day 8. At day 8 of the differentiation program the cells were fed with designated medium supplemented with DMSO, Rosi $(1 \mu \mathrm{M})$ or sample (extract, fractions, falcarinol or falcarindiol). GU bioassay was performed $48 \mathrm{~h}$ later. The cells were then washed first with $200 \mu$ per well PBS ( $\mathrm{pH} 7.2,1 \mathrm{mM} \mathrm{CaCl}_{2}$ and $\left.1 \mathrm{mM} \mathrm{MgSO}_{4}\right)$, subsequently with $200 \mu \mathrm{l}$ per well DMEM $\left(1 \mathrm{~g} \mathrm{~L}^{-1}\right.$ D-glucose) and finally incubated in $200 \mu \mathrm{l}$ per well of the same medium for $2 \mathrm{~h}$ in the incubator at $37{ }^{\circ} \mathrm{C}\left(95 \%\right.$ air and $\left.5 \% \mathrm{CO}_{2}\right)$. Cells were then washed with $200 \mu \mathrm{l}$ per well KRHB ( $\mathrm{pH} \mathrm{7.4)} \mathrm{and}$ incubated with $50 \mu \mathrm{l}$ per well KRHB for $30 \mathrm{~min}$ at $37{ }^{\circ} \mathrm{C}(95 \%$ air and $\left.5 \% \mathrm{CO}_{2}\right)$. Fifty $\mu \mathrm{l}$ per well KRHB containing double amount of the designated concentration of insulin $(10 \mathrm{nM})$ was added, and incubation was continued for exactly $15 \mathrm{~min}$. GU was initiated by the addition of $50 \mu \mathrm{l}$ per well KRHB containing $3.0 \mathrm{mM}$ D-glucose and $0.15 \mu \mathrm{l}$ of $\left[{ }^{14} \mathrm{C}\right] 2$-deoxy-D-glucose (5 mCi $\mathrm{L}^{-1}$ ), yielding a final concentration of $1.0 \mathrm{mM}$ D-glucose. The cells were incubated for exactly $15 \mathrm{~min}$ in the incubator at $37{ }^{\circ} \mathrm{C}\left(95 \%\right.$ air and $\left.5 \% \mathrm{CO}_{2}\right)$. Then $50 \mu \mathrm{l}$ per well Quench buffer (800 mM D-glucose, 50 mM HEPES pH 7.5, $262 \mathrm{mM} \mathrm{NaCl}$ ) were added. The cells were washed three times in $200 \mu \mathrm{l}$ per well ice-cold PBS and lysed in $200 \mu \mathrm{l}$ per well $1 \%$ SDS by shaking for $2 \mathrm{~h}$. Radioactivity in the lysates was determined by scintillation counting. GU was determined in eight parallel wells for crude extract, fractions or pure compounds (falcarinol and falcarindiol) and for each insulin concentration.

\section{Oil Red O staining}

Differentiated 3T3-L1 cells were washed in PBS and fixed with $4 \%$ paraformaldehyde for minimum $1 \mathrm{~h}$ and washed with distilled water. Cells were incubated with Oil Red O solution (8.57 mM Oil Red O in isopropanol) mixed $3: 2$ with water for $30 \mathrm{~min}$ and washed at least twice in water.

\section{PPAR $\gamma$ transactivation bioassay}

Activation of PPAR $\gamma$ elicited by treatment with vehicle $(0.1 \%$ DMSO), positive control ( $1 \mu \mathrm{M}$ Rosi), falcarinol (3, 10 and 30 $\mu \mathrm{M})$ or falcarindiol $(3,10$ and $30 \mu \mathrm{M})$ was determined as described previously. ${ }^{20}$

\section{Quantitative real-time polymerase chain reaction (qPCR)}

Total RNA was isolated on day 10 from MDI treated 3T3-L1 adipocytes (triplicates) using TRIzol reagent. $500 \mathrm{ng}$ of RNA were reverse transcribed with RevertAid (Fermentas, St. LeonRot, Germany) according to the manufacturer's instructions. qPCR analysis was performed using the Mx3000P qPCR system (Agilent Technologies, Denmark) and SYBRGreen JumpStart Taq ReadyMix (Sigma-Aldrich, Denmark). Primers were purchased from Tag Copenhagen A/S (Copenhagen, Denmark). Data was analyzed using the $\Delta \Delta \mathrm{Ct}$ method and gene expression was normalized to TATA box-binding protein. Primers used for analysis of gene expression are shown in Table 1.

\section{Docking studies}

Docking studies were performed with GOLD version 5.1 using default parameters (GoldScore, 100\% search efficiency). ${ }^{21}$ Protein Data Bank entry 2Q5S (human PPAR $\gamma$ ) was selected as protein template. $^{22}$ The active site was determined by selecting all residues within a radius of $6 \AA$ of the co-crystalized ligand for 2Q5S. After docking, all compounds were minimized using LigandScout's general purpose MMFF94 implementation. ${ }^{23}$ The best docking poses for the ligands were selected by developing a 3D pharmacophore with LigandScout. ${ }^{24,25}$ LigandScout was also used for visualization, analysis, and illustrations.

\section{Preparation of myotube cell cultures}

Satellite cells were isolated from semi-membranous muscles of female pigs weighing approx. $12 \mathrm{~kg}$ as described previously, ${ }^{26}$ and stored in liquid nitrogen until used. To prepare myotube 
Table 1 Primer sequences used for analysis of gene expression

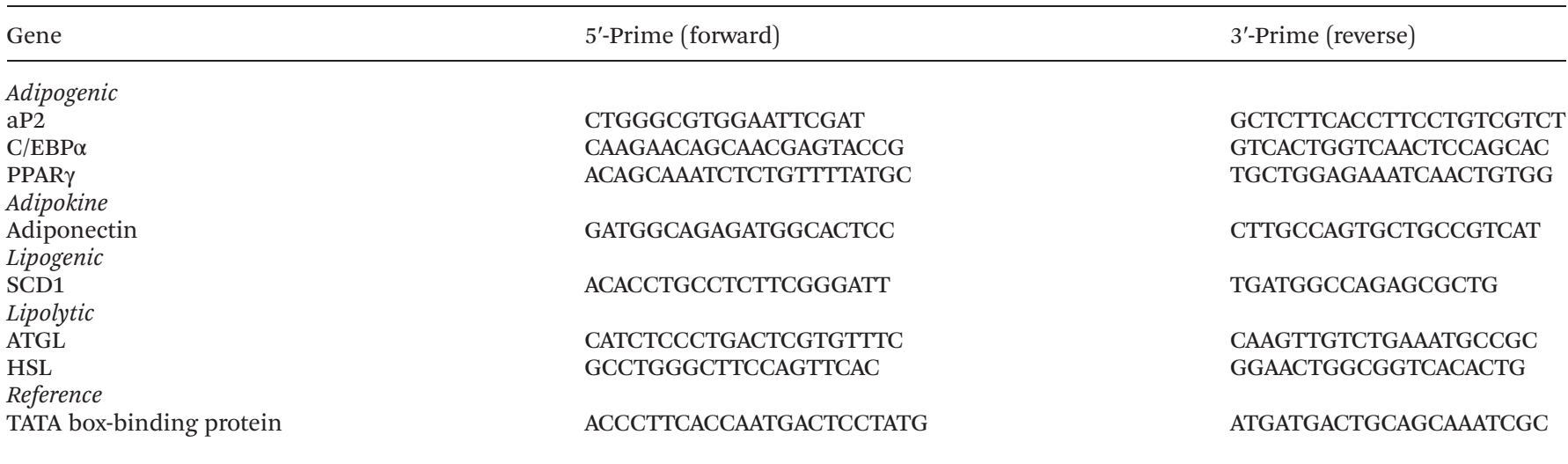

cultures, the cells were seeded on Matrigel matrix coated (1: 50 $\mathrm{v} / \mathrm{v}$ ) in 48-well plates for GU assay. Cells were proliferated in porcine growth medium consisting of $10 \%$ FCS, $10 \%$ horse serum, 80\% DMEM with $25 \mathrm{mM}$ D-glucose and antibiotics $(100$ $\mathrm{IU} \mathrm{ml} \mathrm{m}^{-1}$ penicillin, $100 \mathrm{IU} \mathrm{ml}^{-1}$ streptomycin sulfate, $3 \mu \mathrm{g} \mathrm{ml} \mathrm{m}^{-1}$ amphotericin B, $20 \mu \mathrm{g} \mathrm{ml^{-1 }}$ gentamycin). The cells were grown in porcine growth medium until they reached approximately $80 \%$ confluence in a $\mathrm{CO}_{2}$-related humidified incubator $(95 \%$ air and $5 \% \mathrm{CO}_{2}$ at $37{ }^{\circ} \mathrm{C}$ ). After reaching $80 \%$ confluence cells were allowed to proliferate to confluence in media containing DMEM (7 mM D-glucose), 10\% FCS, and antibiotics for $24 \mathrm{~h}$ and subsequently differentiated into myotubes by incubating with differentiation media (DMEM containing $7 \mathrm{mM}$ D-glucose, $5 \%$ FCS, antibiotics, and $1 \mu \mathrm{M}$ cytosine arabinoside) for at least $48 \mathrm{~h}$.

\section{GU in porcine myotube cell cultures}

Differentiated myotubes were treated with serum free media (DMEM with $7 \mathrm{mM}$-glucose, antibiotics, and $1 \mu \mathrm{M}$ cytosine arabinoside) overnight, followed by incubation with falcarinol or falcarindiol in concentrations of $0.3,1,3,10$ and $30 \mu \mathrm{M}$ with or without insulin $(10 \mathrm{nM})$ for $1 \mathrm{~h}$. Controls received equal volumes of DMSO. The myotubes were then washed thrice with HEPES buffered saline (20 mM HEPES, $140 \mathrm{mM}$ $\mathrm{NaCl}, 5 \mathrm{mM} \mathrm{KCl}, 2.5 \mathrm{mM} \mathrm{MgSO}_{4}, 1 \mathrm{mM} \mathrm{CaCl}_{2}$, adjusted to $\mathrm{pH}$ 7.4 with $2 \mathrm{M} \mathrm{NaOH}$ ) and incubated with $250 \mu \mathrm{l}$ of $0.1 \mathrm{mM}$ 2-deoxy- $\left[{ }^{3} \mathrm{H}\right]$-D-glucose per well for $30 \mathrm{~min}$. Myotubes were then quickly washed thrice with $500 \mu \mathrm{L}$ ice cold PBS per well, and the cells lysed by adding $250 \mu \mathrm{l}$ of $0.05 \mathrm{M} \mathrm{NaOH}\left(37^{\circ} \mathrm{C}\right)$ per well and placed on a shaking board for $30 \mathrm{~min}$. The cell lysate was transferred to a scintillation tube, mixed with scintillation liquid (Ultima Gold, PerkinElmer Inc.) in 1:10 ratio and counted in a Win spectral, 1414 liquid scintillation counter (PerkinElmer). The data was normalized with protein concentration per well. For each experiment, satellite cells from 3 pigs were used, with 6 replicates per pig.

\section{Statistical analysis}

The data on 3T3-L1 adipocytes were analyzed by SAS statistical programming software (Ver. 9.2; SAS Institute Inc., Cary, NC,
USA) and OriginPro software (OriginPro 8.0, OriginLab Corporation). Data were expressed as the mean \pm standard deviation (SD) of three independent experiments in triplicates. The identification of significances between different groups was carried out with Student's $t$-test. A $P$ value $<0.05$ was considered statistically significant. For experiments on porcine myotubes, statistical analysis of data was conducted using the 'Mixed' procedure of SAS statistical programming software (Ver. 9.2; SAS Institute Inc.). The model for myotube cultures consisted of treatments and their interactions as fixed effects, and experiments, replicate determinations, and pigs within treatments as random effects. LSMeans were separated with the P-diff option.

\section{Results and discussion}

Isolation of potentially antidiabetic polyacetylenes by bioassayguided fractionation

The DCM extract of carrot roots was shown to stimulate insulin-dependent GU in 3T3-L1 adipocytes in a dose dependent manner (Fig. 1). Fractionation of the extract by normal phase flash column chromatography resulted in 10 fractions (A-J) of which fraction $\mathrm{C}$ and $\mathrm{F}$ were bioactive showing significant stimulation of insulin dependent $\mathrm{GU}$ relative to the solvent vehicle $(0.1 \%$ DMSO). Fraction $\mathrm{C}$ and $\mathrm{F}$ increased stimulation of insulin-dependent GU with increased concentrations thus indicating a dose-dependent relationship (Fig. 1). Fraction C contained mainly falcarinol and minor amounts of falcarindiol while fraction $\mathrm{F}$ contained mainly falcarindiol as shown by TLC and LC-DAD-MS. In addition, other minor metabolites (less than $5 \%$ ) were detected by LC-DAD-MS in the active fractions, but were not further investigated. Finally, fractionation of fraction $\mathrm{C}$ and $\mathrm{F}$ by semi-preparative HPLC resulted in 14 fractions (C1-C14) and 9 fractions (F1-F9), respectively, of which fraction $\mathrm{C} 6$ and fraction $\mathrm{F} 2$ were found to be bioactive in the insulin-stimulated GU bioassay. Fraction C6 contained falcarinol (10 $\mathrm{mg}$, purity $>98 \%$ ) and fraction $\mathrm{F} 2$ contained falcarindiol (13 mg, purity $>98 \%$ ). The compounds 


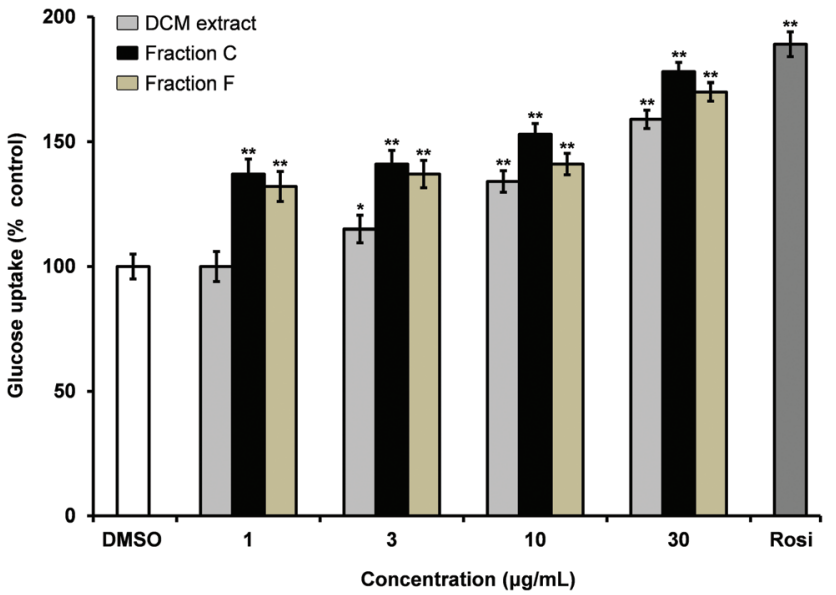

Fig. 1 Effect of the DCM extract of carrot roots and the active fractions $\mathrm{C}$ and $\mathrm{F}$ in concentrations of $1,3,10$ and $30 \mu \mathrm{g} \mathrm{ml}^{-1}$ on insulin-dependent glucose uptake in mature 3T3-L1 adipocytes, relative to the vehicle (100\%). Insulin concentration was $10 \mathrm{nM}$, vehicle $0.1 \% \mathrm{DMSO}$, and the positive control was Rosi $(1 \mu \mathrm{M})$. All values are expressed as mean \pm SD of three independent experiments in triplicates. ${ }^{*} P<0.01$, and ${ }^{* *} P<$ 0.001 indicate significance relative to $0.1 \%$ DMSO.

were identified by LC-DAD-MS, $1 \mathrm{D}$ and 2D NMR, and optical rotation and tested for their potential antidiabetic effects.

Falcarinol and falcarindiol stimulate insulin-dependent GU in a dose dependent manner in adipocytes and enhance PPAR $\gamma$ activity

Falcarinol and falcarindiol were tested for their ability to increase insulin-dependent GU in 3T3-L1 adipocytes at concentrations of $0.3,1,3,10$ and $30 \mu \mathrm{M}$. Falcarindiol showed a significant stimulation of GU at all tested concentrations indi- cating that the stimulation was dose-dependent (Fig. 2A). Falcarinol showed a significant effect in GU at 1,10 and $30 \mu \mathrm{M}$ in a dose-dependent manner (Fig. 2A). Falcarindiol was, however, more potent than falcarinol, at all concentrations tested. Falcarinol and falcarindiol are fatty acid derivatives. Fatty acids and fatty acid derivatives such as prostanoids are well known endogenous activators of PPAR $\gamma$; thus the modes of action of falcarinol and falcarindiol in relation to their antidiabetic potential may be partially due to activation of this nuclear receptor. $^{27}$ This assumption is in accordance with a recent study by Atanasov et al. ${ }^{28}$ who isolated falcarindiol and five other falcarindiol-type polyacetylenes from the DCM extract of the medicinal plant Notopterygium incisum by bioassay-guided fractionation. Atanasov et $a .^{28}$ used a PPAR $\gamma$-driven luciferase reporter model to isolate bioactive polyacetylenes in contrast to an insulin-stimulated GU bioassay used in this study. All polyacetylenes isolated from $N$. incisum activated PPAR $\gamma$ as partial agonists with maximal activation ranging from 1.73- to 3.26-fold compared to a 9.28-fold activation of the positive control pioglitazone. The partial PPAR $\gamma$ agonism was furthermore confirmed by docking experiments. Falcarindiol was in this study also assessed for its effect on basal and insulindependent GU. Falcarindiol showed no effect on basal GU uptake in adipocytes, whereas it showed an effect on insulindependent GU at $10 \mu \mathrm{M}$ in accordance with the results of the present study (Fig. 2).

In the present study falcarindiol elicited a significant activation of PPAR $\gamma$ at all tested concentrations with a 2-fold activation at lower concentrations $(3 \mu \mathrm{M}$ and $10 \mu \mathrm{M})$ and 3 -fold activation, at the highest concentration $(30 \mu \mathrm{M})$ compared to the vehicle $0.1 \%$ DMSO (Fig. 2B). Falcarinol on the other hand showed only a weak but still significant 1.6-fold activation of PPAR $\gamma$ at a concentration of $10 \mu \mathrm{M}$, whereas no significant effect was observed at $3 \mu \mathrm{M}$ and $30 \mu \mathrm{M}$ (Fig. 2B). Thus our
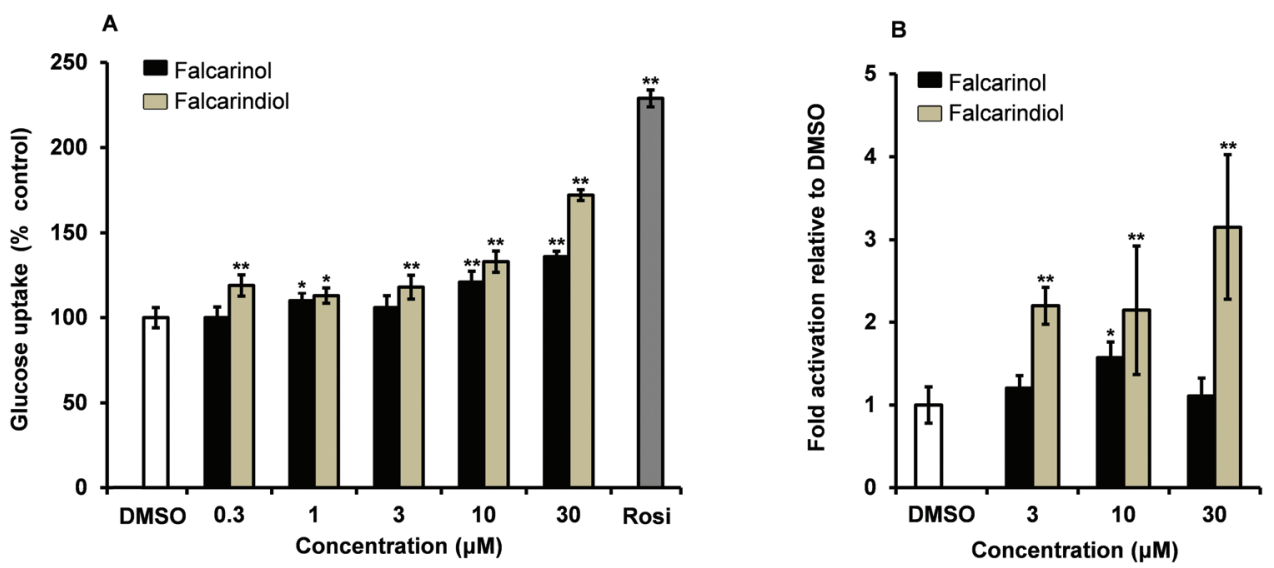

Fig. 2 (A) Effect of falcarinol and falcarindiol in concentrations of $0.3,1,3,10$ and $30 \mu \mathrm{M}$ on insulin-dependent glucose uptake in mature 3T3-L1 adipocytes relative to the vehicle (100\%). Insulin concentration was $10 \mathrm{nM}$, vehicle $0.1 \%$ DMSO, and the positive control was Rosi (1 $\mu \mathrm{M})$. (B) Effect of 3, 10 and $30 \mu \mathrm{M}$ falcarinol and falcarindiol on PPAR $\gamma$-mediated transactivation using a mouse embryonic fibroblast cell line for transfection assays. The vehicle (0.1\% DMSO) was set to 1 and the results were normalized to this. The positive control Rosi was measured to 61 and 86 relative to $0.1 \%$ DMSO in the two PPAR $\gamma$ transactivation assays, respectively. All values are expressed as mean \pm SD of three independent experiments in triplicates. ${ }^{*} P<0.01$, and ${ }^{* *} P<0.001$ indicate significance relative to $0.1 \%$ DMSO. 
PPAR $\gamma$ activity data confirm previous results for falcarindiol ${ }^{28}$ but indicates also that falcarinol can act as a PPAR $\gamma$ agonist, although as a weaker agonist compared to falcarindiol.

\section{Predicted binding modes of falcarinol and falcarindiol in the PPAR $\gamma$ LBD are typical for PPAR $\gamma$ partial agonists}

In order to find an explanation for the different PPAR $\gamma$ activities of falcarinol and falcarindiol we performed docking studies using both polyacetylenes, and compared their binding modes to the PPAR $\gamma$ LBD. The PPAR $\gamma$ LBD contains a large Y-shaped ligand binding cavity consisting of an entrance (arm III) that branches off into two binding pockets (arm I and II). Arm I is the only substantially polar cavity of the PPAR $\gamma$ LBD, whereas arms II and III are mainly hydrophobic. ${ }^{22}$ Docking studies predicted that falcarinol and falcarindiol are able to establish a hydrogen bond to Ser342. The best docking pose for falcarindiol (Fig. 3A and B) shows that the hydrogen bond is established between the hydroxyl group at C-8 while the best docking pose for falcarinol (Fig. 3C and D) predicts a hydrogen bond to the hydroxyl group at C-3. In addition, falcarindiol was able to establish a hydrogen bond between the hydroxyl group at C-3 and Leu228 (Fig. 3B). The hydrophobic contacts between falcarinol and the LBD were with Leu228, Ile281, Ala292, Ile326, Met329, Leu330, Leu333, Ile341, and Met348. For falcarindiol the hydrophobic contacts were with Leu255, Ile281, Met329, Leu333, and Ile341. The extra hydrogen bond of falcarindiol to the LBD of PPAR $\gamma$ compared to fal- carinol suggests a stronger binding to the LBD (Fig. 3). This may explain why falcarindiol exhibited a more significant activation of PPAR $\gamma$ compared to falcarinol.

The predicted binding mode of falcarinol and falcarindiol with arms II and III of the LBD of PPAR $\gamma$ (Fig. 3) are typical for PPAR $\gamma$ partial agonists. ${ }^{22}$ In addition, no hydrogen bond interaction of falcarinol and falcarindiol and residues Ser289, His323, His449 and/or Tyr473 from arm I of the LBD of PPAR $\gamma$ was predicted, which are typical for PPAR $\gamma$ full agonists. ${ }^{29}$ The predicted binding modes of falcarindiol to the PPAR $\gamma$ LBD are in accordance with previous docking experiments, ${ }^{28}$ although another docking model was used in this study.

\section{The effect of falcarinol and falcarindiol on adipocyte differentiation}

The finding that falcarinol and falcarindiol both exhibited characteristics of a PPAR $\gamma$ partial agonist and enhanced insulin-dependent GU in mature adipocytes prompted us to investigate how these compounds affected adipocyte differentiation. When falcarinol and falcarindiol at $30 \mu \mathrm{M}$ concentration were included from day 0 to day 8 of differentiation falcarinol, but not falcarindiol impaired adipocyte differentiation as visualized by Oil Red O staining (Fig. 4). The impairment of MDI-induced adipocyte differentiation by falcarinol may reflect competition between endogenous activators of PPAR $\gamma$ needed for induction of adipocyte differentiation, ${ }^{30}$ and the poor PPAR $\gamma$ activation of falcarinol.
A

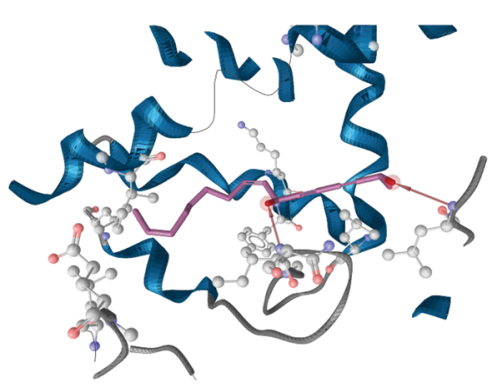

C

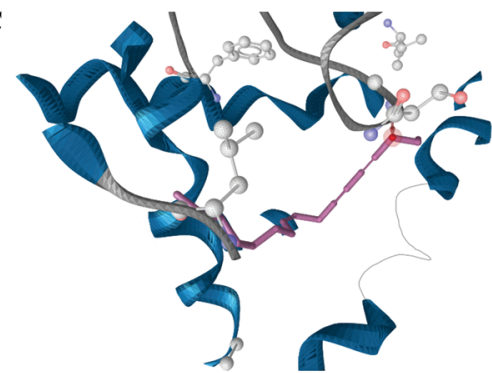

B

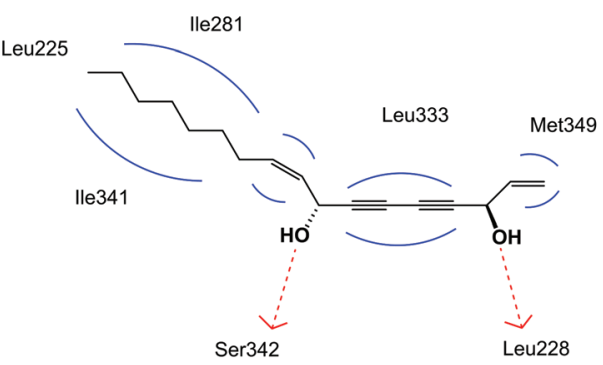

D

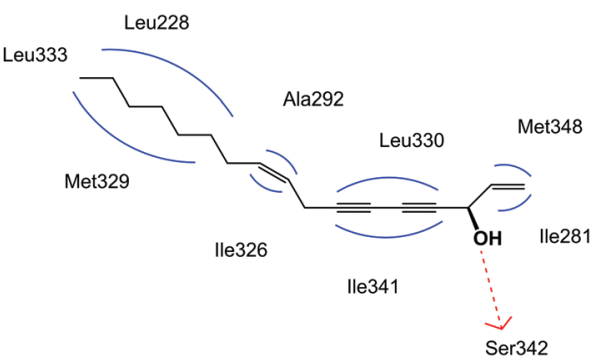

Fig. 3 Predicted binding modes of falcarindiol ( $A$ and $B$ ) and falcarinol ( $C$ and $D)$ shown as 3D and 2D depictions including chemical features of the interaction pattern derived from the docking pose ( $B$ and $D)$. Hydrogen bonds in the 3D depiction are shown as arrows (A and $C)$ and chemical features in the $2 \mathrm{D}$ depictions are color coded: red dashed arrow = hydrogen bond donor; blue line = hydrophobic contact. 


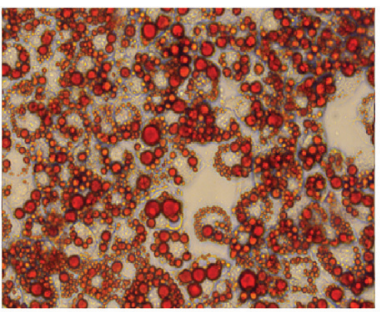

DMSO

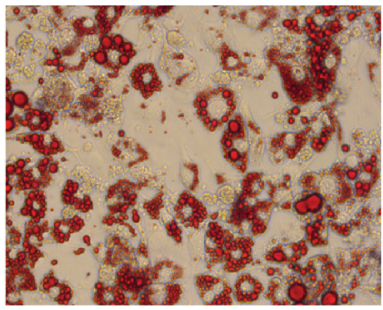

Falcarinol

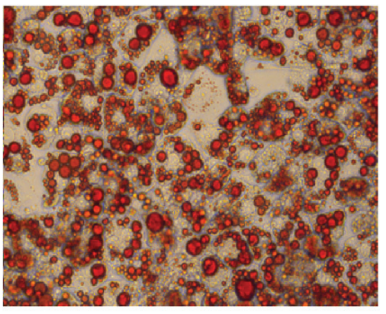

Rosi

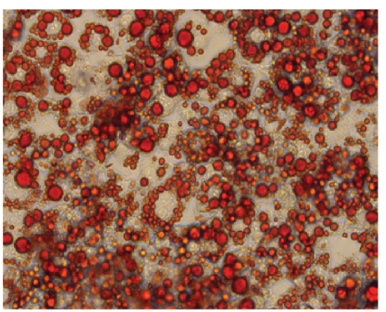

Falcarindiol
Fig. 4 Adipocyte differentiation using the MDI protocol on 3T3-L1 preadipocytes in the continuous presence of $0.1 \%$ DMSO (vehicle), $1 \mu \mathrm{M}$ Rosi, $30 \mu \mathrm{M}$ falcarinol, and $30 \mu \mathrm{M}$ falcarindiol, respectively. The cells were stained on day 8 .

Gene expression during the early phase of adipocyte differentiation of 3T3-L1 adipocytes indicates that falcarinol and falcarindiol have distinct modes of action

Increased expression of adipocyte differentiation markers is associated with insulin sensitivity. ${ }^{31}$ The critical window for ligand-dependent induction of adipocyte differentiation of 3T3-L1 preadipocytes is from day 0 to 4 after induction of differentiation. ${ }^{30}$ To assess the possible mechanisms by which falcarinol and falcarindiol affect the early stages of adipocyte differentiation, we examined the expression of genes involved in adipogenesis [PPAR $\gamma, \mathrm{CCAAT} /$ enhancer-binding protein $\alpha$ $(\mathrm{C} / \mathrm{EBP} \alpha)$ and adipocyte protein 2 (aP2)], lipogenesis [stearoylcoenzyme A desaturase 1 (SCD1)], lipolysis [adipose triglyceride lipase (ATGL) and hormone-sensitive lipase (HSL)] as well as expression of the gene for the adipocyte-derived hormone adiponectin by qPCR (Table 1 ).

Adiponectin has been shown to play an important role in mediating GU in adipocytes and muscle cells, ${ }^{32,33}$ and was significantly up-regulated by addition of falcarinol and falcarindiol (Fig. 5A). This finding fits with the stimulatory effects seen for the insulin-dependent GU in adipocytes for both polyacetylenes (Fig. 2A).

PPAR $\gamma$ and $\mathrm{C} / \mathrm{EBP} \alpha$ are considered "master regulators" of adipogenesis. $^{34}$ No up-regulation of these key markers of adipogenesis was observed for falcarinol and falcarindiol (Fig. 5A), which is in accordance with the two polyacetylenes being weak activators of PPAR $\gamma .{ }^{6,7}$ aP2 also known as fatty acid binding protein 4 (FABP4) is a key mediator of intracellular transport and metabolism of fatty acids and is abundantly
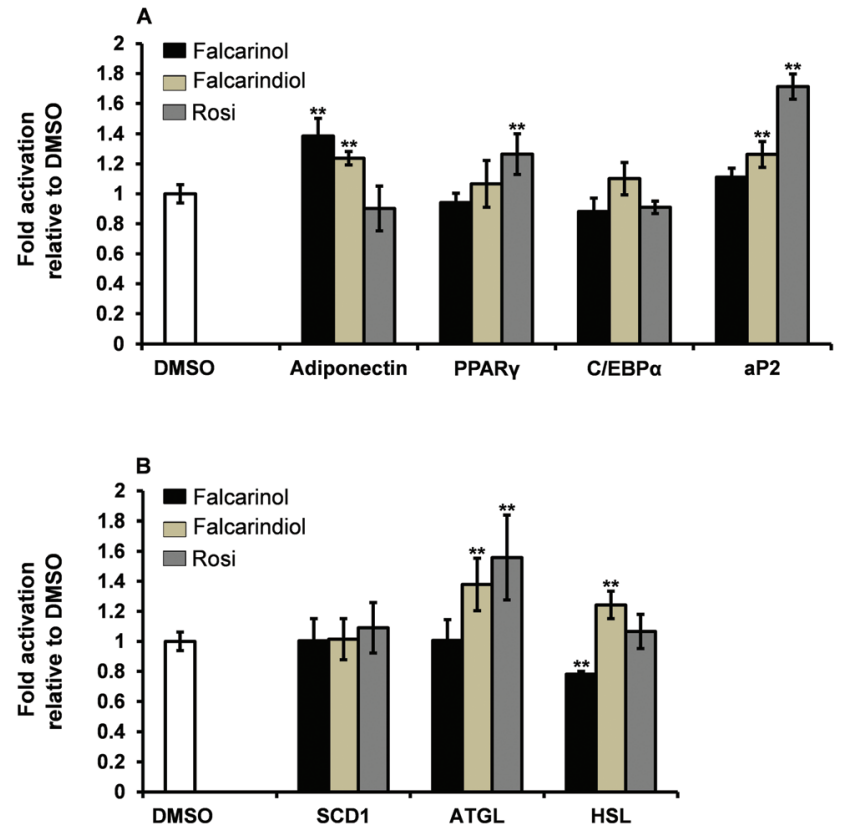

Fig. 5 Effects of falcarinol and falcarindiol on gene expression of adiponectin, PPAR $\gamma, \mathrm{C} / \mathrm{EBP} \alpha$ and aP2 (A) and SCD1, ATGL and HSL (B) in differentiating 3T3-L1 cells. 3T3-L1 preadipocytes were subjected to differentiation according to the MDI protocol in the presence of $30 \mu \mathrm{M}$ falcarinol and $30 \mu \mathrm{M}$ falcarindiol, respectively. The cells were harvested on day 4 of differentiation. $0.1 \%$ DMSO (vehicle) was set to 1 and the results normalized to this value, while Rosi $(1 \mu \mathrm{M})$ was a positive control for full agonist activation of PPAR $\gamma$ for comparison. Data were expressed as the mean $\pm S D$ of three independent experiments in triplicates. ${ }^{*} P<0.001$ indicates significance relative to $0.1 \%$ DMSO.

expressed in adipocytes and may represent as much as 1-3\% of the intracellular soluble proteins. Its expression is highly regulated during adipocyte differentiation through actions of PPAR $\gamma$ and $\mathrm{C} / \mathrm{EBP} \alpha{ }^{35,36}$ Falcarinol was found not to have any significant effect on aP2 gene expression in keeping with it being a relatively poor activator of PPAR $\gamma$ (Fig. 5A). Further, the significant up-regulation of aP2 gene expression in response to treatment with falcarindiol (Fig. 5A) reflected that falcarindiol is more potent and has higher efficacy in relation to PPAR $\gamma$ activation. This finding is in accordance with the results of Atanasov et al. ${ }^{28}$

SCD1 is a key enzyme in the control of lipid metabolism and is rate limiting for the conversion of saturated fatty acids to monounsaturated fatty acids and thus the formation of triacylglycerols and other lipids. ${ }^{37,38}$ Neither of the polyacetylenes affected SCD1 gene expression significantly (Fig. 5B).

ATGL and HSL are the major key enzymes involved in the breakdown of triacylglycerols to fatty acid products. ${ }^{39,40}$ It has been demonstrated that the free acid products released during lipolysis can serve as intrinsic ligands for PPAR $\gamma$ or serve as pro-ligands for PPAR $\gamma \cdot{ }^{39}$ Thus ATGL and HSL can be considered as modulators of adipose metabolism through PPAR $\gamma$. The significant up-regulation of the gene expression for ATGL and HSL when adipocytes were treated with falcarindiol 
(Fig. 5B) is interesting as expression of ATGL in adipocytes has been shown to affect insulin sensitivity and to contribute to the increase of plasma fatty acids. ${ }^{41}$ Excess release of free fatty acids can impair insulin signalling in insulin sensitive tissue and thus further analyses of the effect of falcarindiol in relation to whole body insulin sensitivity is warranted. On the other hand falcarinol significantly down-regulated the expression of HSL gene and had no significant effect on ATGL gene expression (Fig. 5B). Along with its inhibitory effect on adipocyte differentiation this indicates that falcarinol may also be an interesting molecule for further analyses.

The above results indicate that the two bioactive polyacetylenes to some extent may have distinct mechanisms of action in adipocytes. However, it is not possible to conclude whether this is linked to their different affinity to the PPAR $\gamma$ LBD and/ or different expression of genes involved in adipose metabolism based on the present data. Thus it could be interesting to investigate the effect of falcarinol and falcarindiol on a more broad range of transcription factors involved in adipogenesis, recruitment of PPAR $\gamma$ co-activators, and glucose transporters in order to elucidate their possible mechanisms of actions and evaluate their potential antidiabetic effects.

\section{Falcarinol and falcarindiol enhance basal and insulin-} dependent GU in myotubes

Skeletal muscle is the main organ for glucose disposal in the body. We have recently demonstrated that the DCM extract of carrot is able to stimulate insulin-dependent GU in porcine skeletal myotubes; ${ }^{16}$ therefore we used this bioassay as a model for the determination of improved GU in skeletal muscle cells. Testing of falcarinol and falcarindiol in porcine myotubes showed that they were able to enhance insulindependent GU and basal GU (without addition of insulin) in these cells (Fig. 6). In the basal GU bioassay both falcarinol and falcarindiol showed significant and dose-dependent activity at concentrations of $0.3,1,3$, and $10 \mu \mathrm{M}$, whereas in the presence of insulin a dose-dependent activity was only observed between $0.3 \mu \mathrm{M}$ and $3 \mu \mathrm{M}$ for both compounds. When falcarinol and falcarindiol concentrations were elevated to $10 \mu \mathrm{M}$ and $30 \mu \mathrm{M}$ a drop in GU was observed for both compounds in the presence of insulin. The GU was in fact significantly lower for falcarinol at a concentration of $30 \mu \mathrm{M}$ compared to the vehicle (0.1\% DMSO). In the basal GU bioassay a drop in bioactivity was only observed at $30 \mu \mathrm{M}$ for both polyacetylenes (Fig. 6). Falcarinol and falcarindiol have previously been shown to exhibit cytotoxicity in vitro towards various cell types of normal and cancer origin. ${ }^{17}$ The drop in bioactivity at high concentrations of these polyacetylenes, i.e., $10 \mu \mathrm{M}$ and $30 \mu \mathrm{M}$, could be due to a cytotoxic effect. However, the results on the effect of falcarinol on GU are in accordance with a recent study where it was also shown that falcarinol enhance phosphorylation of the TBC1D1 protein in porcine myotubes. ${ }^{42}$ The TBC1D1 protein is highly expressed in skeletal muscle and is involved in the regulation of insulin- and contraction-induced glucose transport, ${ }^{43}$ suggesting that falcarinol is able to enhance translocation of glucose transporter
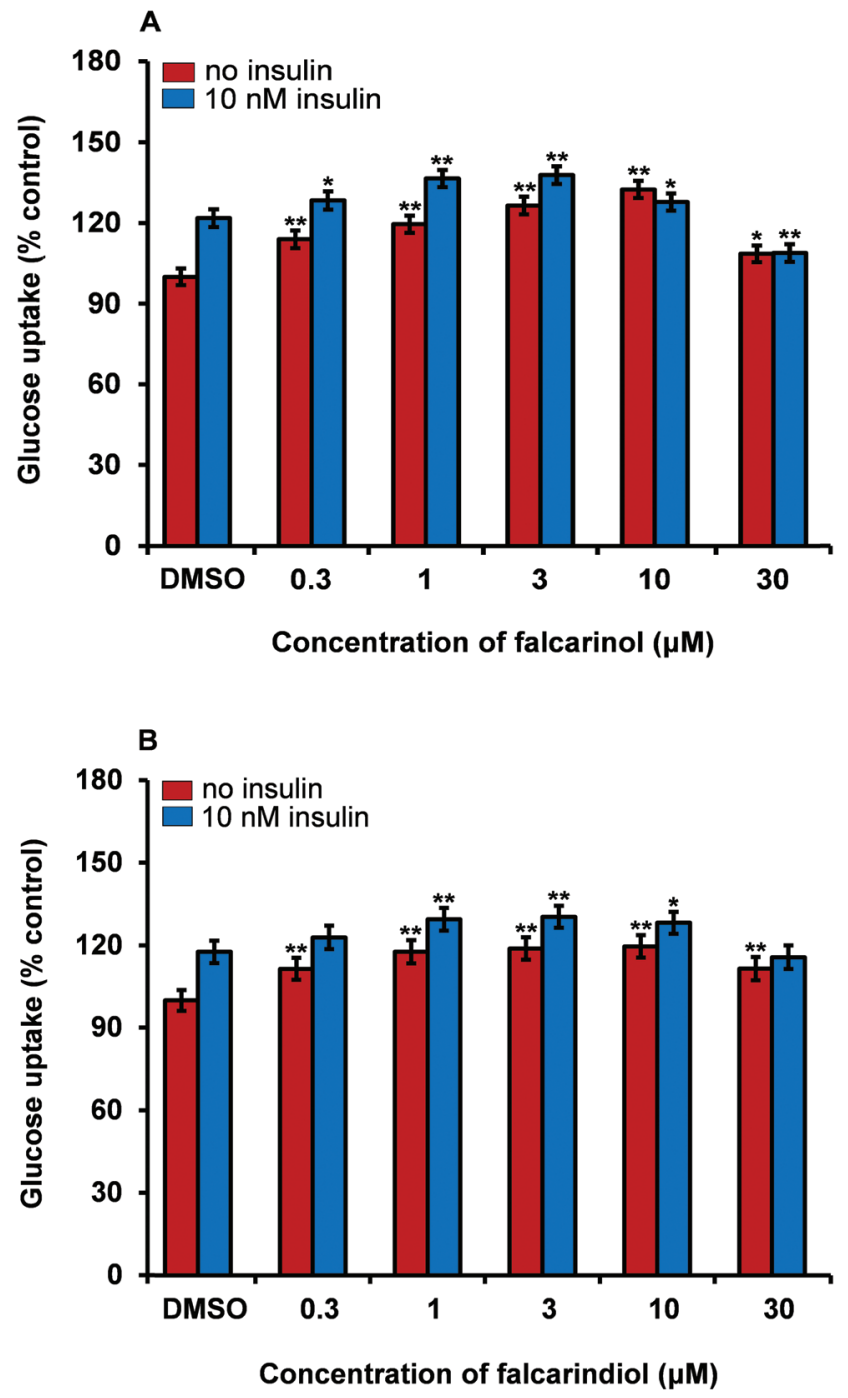

Fig. 6 Effect of falcarinol (A) and falcarindiol (B) in concentrations of $0.3,1,3,10$ and $30 \mu \mathrm{M}$ on insulin-dependent (10 nM) glucose uptake (GU) and basal GU (no insulin) in porcine myotube cultures. GU is given in percent of the vehicle $(0.1 \%$ DMSO), which was set to $100 \%$. The plotted values are least square means \pm SEM. Number of pigs used $=3$ and number of replicates taken per pig $=6$. ${ }^{*} P<0.01$, and ${ }^{* *} P<0.001$ indicate significance relative to $0.1 \%$ DMSO.

type 4 and thereby GU via a TBC1D1-dependent mechanism in skeletal muscles. ${ }^{42}$ The fact that the GU of falcarinol and falcarindiol in porcine myotubes were dose-dependent and almost of the same magnitude in the presence or absence of insulin supports the effects on GU observed in adipocytes, although different mechanisms of action maybe involved.

\section{Conclusions}

A DCM extract of carrots was shown to contain natural products that are able to stimulate GU in vitro in 3T3-L1 adipocytes and porcine myotube cultures. Bioassay-guided 
fractionation of the extract using a GU bioassay resulted in isolation of the bioactive polyacetylenes falcarinol and falcarindiol. The polyacetylenes were shown to enhance GU in both adipocytes and myotubes in a dose-dependent manner and displaying the characteristics of PPAR $\gamma$ partial agonists. Falcarinol was shown to inhibit adipocyte differentiation as evident by gene expression studies and Oil Red O staining, whereas falcarindiol did not inhibit adipocyte differentiation, which indicates that these polyacetylenes have distinct modes of action.

Falcarinol and falcarindiol occur besides in carrots also in many other Apiaceae vegetables such as parsley, celery, parsnip and fennel; ${ }^{44}$ hence, falcarinol and falcarindiol are relatively widespread bioactive food ingredients. Finally, it has been shown that falcarinol and falcarindiol enters the systemic circulation in humans through the intake of carrots. ${ }^{40}$ However, the potential effects of these compounds toward T2D still remain to be demonstrated in vivo in animal and human trials. In conclusion, the slightly different effects of the two polyacetylenes that both enhance GU suggest that they are of interest in relation to prevention and/or treatment of T2D and could serve as interesting scaffolds for novel PPAR $\gamma$ partial agonists with insulin sensitizing properties.

\section{Abbreviations}

$\begin{array}{ll}\text { aP2 } & \text { Adipocyte protein } 2 \\ \text { ATGL } & \text { Adipose triglyceride lipase } \\ \text { C/EBP } \alpha & \text { CCAAT/enhancer-binding protein } \alpha \\ \text { CS } & \text { Calf serum } \\ \text { DCM } & \text { Dichloromethane } \\ \text { FCS } & \text { Foetal calf serum } \\ \text { GU } & \text { Glucose uptake } \\ \text { HSL } & \text { Hormone-sensitive lipase } \\ \text { KRHB } & \text { Krebs-Ringer-Hepes buffer } \\ \text { LBD } & \text { Ligand binding domain } \\ \text { MDI } & \text { 1-Methyl-3-isobutylxanthine, dexamethasone and } \\ & \text { insulin } \\ \text { PPAR } & \text { Peroxisome proliferator-activated receptor } \\ \text { qPCR } & \text { Quantitative real-time polymerase chain reaction } \\ \text { Rosi } & \text { Rosiglitazone } \\ \text { SCD1 } & \text { Stearoyl-coenzyme A desaturase 1 } \\ \text { T2D } & \text { Type 2 diabetes } \\ \text { TZD } & \text { Thiazolidinedione }\end{array}$

\section{Conflict of interest}

The authors declare no conflict of interest.

\section{Acknowledgements}

This work was supported by the Danish Council for Strategic Research (Project number 09-063086).

\section{References}

1 G. Danaei, M. M. Finucane, Y. Lu, G. M. Singh, M. J. Cowan, C. J. Paciorek, J. K. Lin, F. Farzadfar, Y. H. Khang, G. A. Stevens, M. Rao, M. K. Ali, L. M. Riley, C. A. Robinson and M. Ezzati, Lancet, 2011, 378, 31-40.

2 R. E. Gimeno and L. D. Klaman, Curr. Opin. Pharmacol., 2005, 5, 122-128.

3 B. Mlinar, J. Marc, A. Janež and M. Pfeifer, Clin. Chim. Acta, 2007, 375, 20-35.

4 R. A. DeFronzo, Diabetes, 1988, 37, 667-687.

5 A. Y. Cheng and I. G. Fantus, Can. Med. Assoc. J., 2005, 172, 213-226.

6 J. P. Berger, A. E. Petro, K. L. Macnaul, L. J. Kelly, B. B. Zhang, K. Richards, A. Elbrecht, B. A. Johnson, G. Zhou, T. W. Doebber, C. Biswas, M. Parikh, N. Sharma, M. R. Tanen, G. M. Thompson, J. Ventre, A. D. Adams, R. Mosley, R. S. Surwit and D. E. Moller, Mol. Endocrinol., 2003, 17, 662-676.

7 A. Zieleniak, M. Wójcik and L. A. Woźniak, Arch. Immunol. Ther. Exp., 2008, 56, 331-345.

8 L. C. Poulsen, M. Siersbæk and S. Mandrup, Semin. Cell Dev. Biol., 2012, 23, 631-639.

9 E. D. Rosen and B. M. Spiegelman, Annu. Rev. Cell Dev. Biol., 2000, 16, 145-171.

10 B. Cariou, B. Charbonnel and B. Staels, Trends Endocrinol. Metab., 2012, 23, 205-215.

11 J. B. Bruning, M. J. Chalmers, S. Prasad, S. A. Busby, T. M. Kamenecka, Y. He, K. W. Nettles and P. R. Griffin, Structure, 2007, 15, 1258-1271.

12 V. Zoete, A. Grosdidier and O. Michielin, Biochim. Biophys. Acta, 2007, 1771, 915-925.

13 F. Lizcano and D. Vargas, Genet. Mol. Biol., 2013, 36, 134139.

14 R. J. Marles and N. R. Farnsworth, Phytomedicine, 1995, 2, 137-189.

15 G. Y. Yeh, D. M. Eisenberg, T. J. Kaptchuk and R. S. Phillips, Diabetes Care, 2003, 26, 1277-1294.

16 R. B. El-Houri, D. Kotowska, L. C. B. Olsen, S. Bhattacharya, L. P. Christensen, K. Grevsen, N. Oksbjerg, N. Færgeman, K. Kristiansen and K. B. Christensen, Evidence-Based Complementary Alternat. Med., 2014, 2014, 156398.

17 S. Purup, E. Larsen and L. P. Christensen, J. Agric. Food Chem., 2009, 57, 8290-8296.

18 A. Czepa and T. Hofmann, J. Agric. Food Chem., 2003, 51, 3865-3873.

19 M. W. Bernart, J. H. Cardellina II, M. S. Balaschak, M. R. Alexander, R. H. Shoemaker and M. R. Boyd, J. Nat. Prod., 1996, 59, 748-753.

20 K. B. Christensen, R. K. Petersen, S. Petersen, K. Kristiansen and L. P. Christensen, J. Nat. Prod., 2009, 72, 933-937.

21 G. Jones, P. Willett and R. C. Glen, J. Mol. Biol., 1995, 245, 43-53. 
22 L. Guasch, E. Sala, C. Valls, M. Blay, M. Mulero, L. Arola, G. Pujadas and S. Garcia-Vallvé, J. Comput. Aided Mol. Des., 2011, 25, 717-728.

23 T. A. Halgren, J. Comput. Chem., 1996, 17, 490-519.

24 T. Seidel, G. Ibis, F. Bendix and G. Wolber, Drug Discovery Today: Technol., 2010, 7, e221-e228.

25 G. Wolber, A. A. Dornhofer and T. Langer, J. Comput. Aided Mol. Des., 2006, 20, 773-788.

26 P. K. Theil, I. L. Sorensen, P. M. Nissen and N. Oksbjerg, Anim. Sci. J., 2006, 77, 330-337.

27 B. Desvergne and W. Wahli, Endocr. Rev., 1999, 20, 649688.

28 A. G. Atanasov, M. Blunder, N. Fakhrudin, X. Liu, S. M. Noha, C. Malainer, M. P. Kramer, A. Cocic, O. Kunert, A. Schinkovitz, E. H. Heiss, D. Schuster, V. M. Dirsch and R. Bauer, PLoS One, 2013, 8, e61755.

29 A. Farce, N. Renault and P. Chavatte, Curr. Med. Chem., 2009, 16, 1768-1789.

30 P. Hallenborg, C. Jørgensen, R. K. Petersen, S. Feddersen, P. Araujo, P. Markt, T. Langer, G. Furstenberger, P. Krieg, A. Koppen, E. Kalkhoven, L. Madsen and K. Kristiansen, Mol. Cell. Biol., 2010, 30, 4077-4091.

31 T. McLaughlin, A. Sherman, P. Tsao, O. Gonzalez, G. Yee, C. Lamendola, G. M. Reaven and S. W. Cushman, Diabetologia, 2007, 50, 1707-1715.

32 L. Zhao, W. Chai, Z. Fu, Z. Dong, K. W. Aylor, E. J. Barrett, W. Cao and Z. Liu, Circ. Res., 2013, 112, 1263-1271.

33 T. Yamauchi, J. Kamon, H. Waki, Y. Terauchi, N. Kubota, K. Hara, Y. Mori, T. Ide, K. Murakami, N. Tsuboyama-
Kasaoka, O. Ezaki, Y. Akanuma, O. Gavrilova, C. Vinson, M. L. Reitman, H. Kagechika, K. Shudo, M. Yoda, Y. Nakano, K. Tobe, R. Nagai, S. Kimura, M. Tomita, P. Froguel and T. Kadowaki, Nat. Med., 2001, 7, 941-946.

34 E. D. Rosen, C. J. Walkey, P. Puigserver and B. M. Spiegelman, Genes Dev., 2000, 14, 1293-1307.

35 L. Sun, A. C. Nicholson, D. P. Hajjar, A. M. Gotto Jr. and J. Han, J. Lipid Res., 2003, 44, 1877-1886.

36 T. Shan, W. Liu and S. Kuang, FASEB J., 2013, 27, 277-287.

37 A. Yao-Borengasser, N. Rassouli, V. Varma, A. M. Bodles, N. Rasouli, R. Unal, B. Phanavanh, G. Ranganathan, R. E. McGehee Jr. and P. A. Kern, J. Clin. Endocrinol. Metab., 2008, 93, 4431-4439.

38 J. C. Ralston, F. Badoud, B. Cattrysse, P. D. McNicholas and D. M. Mutch, Int. J. Obesity, 2014, 38, 1449-1456.

39 W. J. Shen, Z. Yu, S. Patel, D. Jue, L. F. Liu and F. B. Kraemer, Biochim. Biophys. Acta, 2011, 1811, 9-16.

40 M. Rogne and K. Taskén, Horm. Metab. Res., 2014, 46, 833840.

41 E. E. Kershaw, J. K. Hamm, L. A. W. Verhagen, O. Peroni, M. Katic and J. S. Flier, Diabetes, 2006, 55, 148-157.

42 S. Bhattacharya, M. K. Rasmussen, L. P. Christensen, J. F. Young, K. Kristiansen and N. Oksbjerg, J. Biochem. Pharmacol. Res., 2014, 2, 91-98.

43 D. An, T. Toyoda, E. B. Taylor, H. Yu, N. Fujii, M. F. Hirshman and L. J. Goodyear, Diabetes, 2010, 59, 1358-1365.

44 L. P. Christensen and K. Brandt, J. Pharm. Biomed. Anal., 2006, 41, 683-693. 